\title{
Planarians: A Versatile and Powerful Model System for Molecular Studies of Regeneration, Adult Stem Cell Regulation, Aging, and Behavior
}

\author{
Néstor J. Oviedo, Cindy L. Nicolas, Dany S. Adams, and Michael Levin ${ }^{1}$ \\ Center for Regenerative and Developmental Biology, Forsyth Institute and Developmental Biology Department, Harvard School \\ of Dental Medicine, Boston, MA 02115, USA
}

\section{INTRODUCTION}

In recent years, planarians have been increasingly recognized as an emerging model organism amenable to molecular genetic techniques aimed at understanding complex biological tasks commonly observed among metazoans. Growing evidence suggests that this model organism is uniquely poised to inform us about the mechanisms of tissue regeneration, stem cell regulation, tissue turnover, pharmacological action of diverse drugs, cancer, and aging. This article provides an overview of the planarian model system with special attention to the species Schmidtea mediterranea. Additionally, information is provided about the most popular use of this organism, together with modern genomic resources and technical approaches.

\section{RELATED INFORMATION}

A detailed protocol for establishing and maintaining planarian colonies for diverse purposes is described in Establishing and Maintaining a Colony of Planarians (Oviedo et al. 2008a). Details on RNA interference (RNAi), increasingly used to study gene function in planarians, are presented in Gene Knockdown in Planarians Using RNA Interference (Oviedo et al. 2008b). The protocol Live Imaging of Planarian Membrane Potential Using DiBAC ${ }_{4}$ (3) (Oviedo et al. 2008c) illustrates why planarians are suitable for the study of complex physiological processes during regeneration and tissue turnover, in real time. The American Society for Cell Biology library of seminars, iBioSeminars (http://www.ascb.org/ibioseminars/sanchez/sanchez1.cfm), features a lecture on the basics of planarian regeneration.

The Genome Sequence Center at Washington University performed the sequencing of the $S$. mediterranea genome that is currently being assembled. Additional details on the sequencing process can be accessed at http://genome.wustl.edu/, and the final assembly will be posted there for download. The National Center for Biotechnology Information (http://www.ncbi.nlm.nih.gov/) displays trace files generated during the sequencing of the $S$. mediterranea genome and will include expressed sequence tag (EST) data generated during the final assembly of the planarian genome. The $S$. mediterranea Genome Database (SmedGD; http://smedgd.neuro.utah.edu/) integrates all available data associated with the $S$. mediterranea genome, including predicted and annotated genes, ESTs, protein homologies, gene expression patterns, and RNAi phenotypes. An accessible portal (http://planarian.bio.keio.ac.jp/go/EST/P/GO0007582/GO0006915.html) from the Department of Biosciences and Informatics at Keio University (Japan) allows for the search and retrieval of a large collection of Dugesia ryukyuensis ESTs. 


\section{BACKGROUND INFORMATION}

Planarians, free-living nonparasitic invertebrates, are one of the most basal triploblastic organisms, with derivatives of all three germ layers (ectoderm, mesoderm, and endoderm). They represent a critical breakthrough in the evolution of the animal body plan, have bilateral symmetry and encephalization, and are capable of detecting environmental stimuli very efficiently. Planarians are perhaps better known for their extraordinary regenerative capacity, which is associated with a large population of adult stem cells (neoblasts).

An extensive amount of literature on planarian research has accumulated during more than 200 years (Reddien and Sánchez Alvarado 2004). In the last century, several laboratories around the world have used planarians as a biological model in a surprisingly broad and extensive range of applications. Systematic research on planarians was first substantiated by Thomas Hunt Morgan and Harriet Randolph, who studied regeneration in planarians (Randolph 1897; Morgan 1898). The fact that freshwater planarians are commonly found in the wild (e.g., ponds, streams, and rivers) and are easily reared and maintained under laboratory conditions, together with their astonishing regenerative capacity and adult tissue plasticity, were factors that undoubtedly made these organisms a favorite subject for researchers in subsequent years.

Although several hundred planarian species exist, research on freshwater planarians in the last century has been restricted to species either commonly found in the wild or commercially available (mostly members of the Dugesiidae family, including the genera Dugesia, Girardia, Neppia, Romankenkius, Schmidtea, and Spathula). Modern research on freshwater planarians has applied diverse state-of-the-art techniques to understand their cellular biology, genetics, genomics, behavior, and other aspects at the molecular level (Sánchez Alvarado 2006; Oviedo and Levin 2008).

In the last 10 years, attention to planarian molecular and genetic research has been greatly enhanced and has focused on work in two species in which clonal lines have been derived: $S$. mediterranea and Dugesia japonica. The advantages of using S. mediterranea over other planarian species for molecular studies have been reviewed elsewhere (Newmark and Sánchez Alvarado 2002; Sánchez Alvarado 2006), and those advantages have been enhanced with the recent introduction of high-throughput molecular tools, large collections of publicly available DNA sequences, automated systems for the analysis of behavior, and genomic resources (Sánchez Alvarado et al. 2002; Zayas et al. 2005; Hicks et al. 2006; Robb et al. 2007). Although most protocols and techniques can be adapted to different planarian species, here we focus on S. mediterranea, an increasingly popular planarian species used to study the molecular basis of tissue regeneration and stem cell biology. The taxonomic classification for S. mediterranea may follow this order: domain Eukaryota; kingdom Animalia; phylum Platyhelminthes (flatworms); class Turbellaria; order Seriata; suborder Tricladida; family Dugesiidae; genus Schmidtea; species S. mediterranea. Further taxonomic details for the class Turbellaria can be found in the Turbellarian taxonomic database at http://turbellaria.umaine.edu.

About 40 years ago, in an artificial pond at Montjuïc near Barcelona, Spain, Baguñà and Ballester identified the asexual (fissiparous) biotype of Dugesia lugubris s.l. (Benazzi et al. 1970, 1972). Karyological and other studies confirmed that this asexual worm matched a diploid $(2 n=8)$ sexual form of $D$. lugubris s.l. that was known from the Mediterranean islands of Corsica, Sardinia, and Sicily; these two forms were later classified together as D. mediterranea (Benazzi et al. 1975). Further analyses by different investigators led to the renaming of this species as it is currently known: Schmidtea mediterranea (Baguñà 1999). The primary difference between the asexual and sexual strains of $S$. mediterranea is a chromosomal translocation between Chromosomes 1 and 3 that is observed in fissiparous animals only (Baguñà 1999; Newmark and Sánchez Alvarado 2002). The diploid asexual strain of $S$. mediterranea reproduces by transverse fission (i.e., the posterior end attaches to a surface, whereas the anterior part tears away until the animal breaks transversally in the post-pharyngeal region), and the missing structures in each fragment are regenerated in $\sim 1 \mathrm{wk}$. The S. mediterranea sexual strain is a hermaphrodite but requires cross-fertilization in order to lay cocoons that after a few weeks hatch, giving rise to one or several planarians (Sánchez Alvarado 2003). Studies on the geographical distribution of S. mediterranea revealed that the natural habitat of the asexual biotype is limited to the Iberian peninsula and Mallorca, whereas the sexual strain is more widely distributed throughout the islands of the western Mediterranean (De Vries 1985).

In the mid 1990s, Sánchez Alvarado chose planarians as a convenient model in which to study the molecular basis of tissue regeneration (Sánchez Alvarado 2006). Together with Philip Newmark, Sánchez Alvarado selected S. mediterranea because of its extraordinary regenerative properties, karyological features (stable diploid), and relatively small genome size compared to 
other common planarians (Newmark and Sánchez Alvarado 2002; Sánchez Alvarado 2006). Asexual specimens of S. mediterranea were first introduced to the United States by Newmark in 1997; however, those animals did not survive. Then, after an expedition to Montjuïc in 1998, Newmark and Sánchez Alvarado brought to the United States several specimens of fissiparous S. mediterranea that, after several rounds of culture optimization, were successfully reared under laboratory conditions. Some of the specimens that thrived in the new laboratory conditions were individually cut and grown separately to establish clonal lines. This led to the establishment of the asexual strain CIW4 (Carnegie Institution of Washington clonal line 4) (Sánchez Alvarado 2006).

Maria Pala (of Italy) provided the Sánchez laboratory with wild-type animals of the S. mediterranea sexual strain from the Mediterranean island of Corsica (Sánchez Alvarado 2003). Clonal lines were created using a strategy similar to that used to establish CIW4. Laboratory conditions were adjusted to allow sexual reproduction of these clonal worms (Sánchez Alvarado 2003, 2006). This offered a unique opportunity to study embryonic development and functional germ-cell specification in S. mediterranea (Sánchez Alvarado 2003). Furthermore, the inbreeding of these animals (performed by Peter W. Reddien) created "more genetically uniform worms" and led to the generation of the S2F2 line, the source of animals used to sequence the $S$. mediterranea genome.

\section{SOURCES AND HUSBANDRY}

Establishing and Maintaining a Colony of Planarians (Oviedo et al. 2008a) describes in detail how to establish and maintain a colony of planarians for behavioral and other experiments.

\section{Related Species}

Besides S. mediterranea, another planarian species commonly used to study regenerative biology is $D$. japonica. Mixoploid and diploid clonal lines (GI, HI, and SSP) of this organism originated in Japan and were subsequently expanded, mainly in the Watanabe and Agata laboratories. Interestingly, different procedures (e.g., decreasing the temperature of the culture and feeding asexual-state planarians with freeze-thawed sexual-state worms) are commonly used to induce gonad development in asexual worms (Ogawa et al. 1998). Similar to the sexual strain of S. mediterranea, germ cells are fully developed in adult $D$. japonica.

Important advances have been made possible by using D. japonica; in fact, several protocols (e.g., whole-mount in situ hybridization and neoblast purification with flow cytometry) were initially developed in this species and later optimized and adjusted for S. mediterranea (Umesono et al. 1997; Sánchez Alvarado et al. 2002; Hayashi et al. 2006; Sánchez Alvarado 2007). However, there are some important differences between S. mediterranea and D. japonica (including expression of the piwi and innexin genes and sensitivity to drugs) (Nogi and Levin 2005; Reddien et al. 2005; Rossi et al. 2006; Oviedo and Levin 2007). Recently, a D. japonica database with neoblast-associated genes has been published (Rossi et al. 2007b).

\section{USES OF THE PLANARIAN MODEL SYSTEM}

For the last century, planarians have been a favorite model system in many research areas. Not only are planarians easy to rear under laboratory conditions and relatively inexpensive to maintain, they are amenable to pharmacological, behavioral, physiological, molecular genetic, and classical surgical techniques. Thus, in recent years, they have become a popular model for state-of-the-art studies in regenerative biology as well as a low-cost system to produce fast experimental results for classroom science projects and other educational purposes (Oviedo and Levin 2008).

\section{Tissue Regeneration, Cellular Turnover, and Aging}

The fact that adult planarians can regenerate large parts of their bodies from tiny fragments (in $\sim 1 \mathrm{wk}$ ) makes them an outstanding model to study regeneration and morphogenesis in complex tissues (Newmark and Sánchez Alvarado 2002; Agata 2003; Reddien and Sánchez Alvarado 2004). The planarian central nervous system (CNS) is the organ system that has been more extensively studied (Cebrià 2007). The planarian CNS is particularly important because it exhibits evolutionary conservation with higher organisms in many neural receptors (e.g., acetylcholine receptor, fibroblast growth factor receptor, and netrin receptor), neuroactive molecules (e.g., $\gamma$-aminobutyric acid and 
FMRFamide-like peptides), and axon guidance molecules (e.g., netrin and roundabout receptor-robo). Recent functional/behavioral assays suggest that this conservation holds at the structural and physiological levels (for references, see Oviedo and Levin 2008). Moreover, the molecular conservation of the CNS also indicates that planarians are a good model in which to test the effects and mechanisms of drugs using large pharmacological screens (Oviedo and Levin 2008).

Remarkably, the rapid reconstruction of multiple missing parts (e.g., CNS, muscle, digestive, sensory system, and epithelium) in S. mediterranea is accompanied by an adjustment in growth to accommodate a new body size. In other words, small planarian fragments regenerate structures such as the CNS with proportions that are based on the new size of the animal, rather than the size of the original fragment (Newmark and Sánchez Alvarado 2002; Oviedo et al. 2003; Reddien and Sánchez Alvarado 2004). Additionally, intact adult animals have the capacity to regulate their body sizes according to metabolic status (i.e., worms grow when food is available, and, if starved, they reduce their size proportionally in a process known as "degrowth"). Interestingly, both growth and degrowth in S. mediterranea involve well-regulated mechanisms for adding and subtracting cells in the body (Baguñà and Romero 1981; Oviedo et al. 2003). Taken together, these observations indicate that planarians possess efficient mechanisms for synchronizing metabolic status and tissue maintenance. The aging process in planarians is fascinating because the neoblasts' progeny regularly replaces senescent cells in different organs. Thus, their tissues are continuously renewed. Therefore, $S$. mediterranea is an exquisite model for studying the regulation of adult differentiated tissue remodeling, tissue maintenance, cellular turnover, and aging (Pellettieri and Sánchez Alvarado 2007).

The anatomy of the reproductive system of $S$. mediterranea, which consists of paired ovaries, many testicles, and a copulatory apparatus, has been reviewed elsewhere (Newmark and Sánchez Alvarado 2002). Unlike other commonly studied invertebrate models, planarians do not appear to segregate their germ-cell lineage during early embryogenesis; instead, the reproductive system in sexual planarians has been proposed to be determined by epigenetic mechanisms after hatching (post-embryonically) (Zayas et al. 2005; Sato et al. 2006; Handberg-Thorsager and Saló 2007; Wang et al. 2007). If a planarian with fully developed gonads is starved for several weeks, the gonads are resorbed, and if the same animal is fed and allowed to reach an appropriate size, the reproductive organs are properly regenerated (Newmark and Sánchez Alvarado 2002). Similarly, if the head of a mature sexual planarian is removed, the remaining gonads in the body are resorbed and will develop again only after the head regenerates completely (Newmark and Sánchez Alvarado 2002; Wang et al. 2007).

\section{Stem Cells and Germ Cells}

In planarians, tissue regeneration, germ-cell specification, tissue remodeling, and adult tissue maintenance involve a large population of undifferentiated cells known as neoblasts (planarian stem cells), located throughout the body, which constantly divide. During regeneration, these cells are the source of new tissue. In asexual worms, neoblasts are the only known cell with mitotic activity and are therefore the sole source of new cells. Thus, neoblasts coexist in a microenvironment that is tightly regulated and allows them to respond to signals to self-renew, proliferate, and migrate, giving rise to differentiated progeny that are properly incorporated into demanding tissues. In recent years, an important focus of research in planarians has been centered on understanding, at the genetic and biochemical levels, how neoblasts are regulated in vivo to respond to demanding signals while renewing themselves (Agata 2003; Sánchez Alvarado and Kang 2005; Sánchez Alvarado 2006; Rossi et al. 2007a).

The complexity of the neoblast population is far from being understood (Rossi et al. 2007a). Neoblast populations are mainly recognized by their morphology, spatial distribution, sensitivity to $\gamma$-irradiation, ultrastructural composition, and gene expression patterns (Orii et al. 2005; Reddien et al. 2005; Hayashi et al. 2006; Sato et al. 2006; Handberg-Thorsager and Saló 2007; Higuchi et al. 2007; Oviedo and Levin 2007; Rossi et al. 2007a,b; Wang et al. 2007). Study of the molecular conservation of regulatory molecules (e.g., the piwi genes) in neoblasts has expanded our knowledge of the evolution of stem cells (Sánchez Alvarado and Kang 2005). Furthermore, the identification and characterization of stem cell regulatory molecules that are conserved between vertebrates and planarians (but absent in other classical invertebrate model organisms such as Drosophila melanogaster and Caenorhabditis elegans) is now possible in S. mediterranea (Oviedo et al. 2008d).

The molecular basis of germ-cell specification in adult stages is poorly understood at this time (Zayas et al. 2005). Interestingly, it has been proposed that germ cells in sexual S. mediterranea are 
epigenetically specified at adult stages and that the maintenance of these epigenetic marks can be affected by diverse factors such as metabolic status, regeneration, and seasonal conditions (Zayas et al. 2005). The plasticity of germ-cell specification and maintenance in adult S. mediterranea provides a unique opportunity to investigate regulatory cues that modulate this process. Taking advantage of these properties, the Newmark lab at the University of Illinois used clonal animals of the sexual strain of S. mediterranea to build an EST database comprising two different developmental stages (Zayas et al. 2005). Thus, this database will be useful for the identification of germ-cell markers as well as signaling pathways involved in germ-cell determination and tissue regeneration.

\section{Memory, Learning, and Behavior}

As the simplest animal with a bilaterally symmetrical CNS composed of neurons similar to our own (Cebrià 2007), planarians offer an opportunity to study neural patterning and behavior while working with an easily maintained organism that is highly tractable to pharmacological, surgical, and molecular genetic manipulations. Planarians have developed sensory capabilities for the detection of light, chemical gradients, vibration, electric fields, magnetic fields, and weak $\gamma$-radiation. When proper procedures are followed, the data conclusively show that planarians can learn. Thus, planarians are a unique model system in which memory and regeneration can be studied in the same animal. This kind of system provides an ideal context in which to characterize the impact of naïve stem cells on memories stored in an adult brain, which is of relevance to the current approaches in treating degenerative diseases of the human brain (McKay 2004), or to dissect the mechanisms by which existing memories can be imposed on regenerated brain tissue. For an in-depth discussion of memory and learning in planarians, consult Nicolas et al. (2008).

\section{GENETICS AND GENOMICS RESOURCES}

At this point in time, genetic tools for gain-of-function and permanent genomic modifications in planarians are not well established. Recently, a proposal involving guidelines for gene and protein nomenclature in the planarian species S. mediterranea was made public (Reddien et al. 2008). Applying these nomenclature guidelines will help to standardize naming and facilitate the identification of gene homology and experimental treatments (e.g., RNAi). Briefly, the prefix "Smed" should be attached to the beginning of all gene names; the gene name should be selected to match the convention of the homologous gene and should be lowercase and italicized (e.g., Smed-genex), and when possible, gene names should try to match ortology. In addition, if there exist paralogs for a given gene, the respective planarian gene gets a numerical suffix, not a letter, at the end (e.g., Smed-genex-1). The protein name should be uppercase and not italicized (e.g., SMED-GENEX). Single RNAi should be denoted as follows: Smed-genex(RNAi). If multiple RNAis are used, a semicolon should connect the names: Smed-genex(RNAi); Smed-geney(RNAi). Note that because mutant alleles and transgenes are not yet commonly used in planarian experiments, there is no standard nomenclature for such. For additional details, see Reddien et al. (2008) and http://smedgd.neuro.utah.edu.

The S. mediterranea sexual strain S2F2, which originated in the Sánchez lab, was used for genomic sequencing at the Washington University Genome Sequencing Center (http://genome.wustl.edu/). The S. mediterranea genome database (SmedGD) at http://smedgd.neuro.utah.edu/ includes details about the available DNA sequences, as well as predicted and annotated genes, ESTs, protein homologies, gene expression patterns, and RNAi phenotypes (Robb et al. 2007). Complementary information on a large collection of ESTs from sexual and asexual $S$. mediterranea is publicly available from the laboratories of Newmark and Sánchez Alvarado (Sánchez Alvarado et al. 2002; Zayas et al. 2005).

\section{TECHNICAL APPROACHES}

Many published protocols from other well-established invertebrate systems such as $D$. melanogaster and $C$. elegans are quite robust and can be adapted for planarians to produce results in a short time ( $<1 \mathrm{wk}$ ). Basic molecular approaches include purification of mRNA to evaluate gene expression (both quantitatively and qualitatively), antibody staining to evaluate the spatial distribution of molecular markers and morphogenesis during regeneration, in situ hybridization to evaluate the spatial distribution of messenger RNA, and quantitative real-time-polymerase chain reaction (RT-PCR) to evaluate transcript levels in planarian tissue. One common procedure to evaluate whether a specific transcript is associated with neoblast cells is to treat some worms with $\gamma$ irradiation (which eliminates neoblasts) 
and then compare the patterns and/or levels of gene expression between irradiated and untreated worms.

Methods used successfully for developmental studies include tissue fixation for immunostaining, in situ hybridization, and gene knockdown with RNAi. The latter is most commonly applied by microinjecting double-stranded RNA (dsRNA) as described in Gene Knockdown in Planarians Using RNA Interference (Oviedo et al. 2008b) or by feeding the worms with artificial food carrying modified bacteria. Neoblasts are the only known proliferative cell in the worm, so labeling with the thymidine analog bromodeoxyuridine (BrdU) (Newmark and Sánchez Alvarado 2000) can be used to evaluate the cell cycle and the migration and integration of the neoblasts into differentiated tissues. Neoblast subpopulations can be isolated by flow cytometry (fluorescence-activated cell sorting [FACS]) (Reddien et al. 2005; Hayashi et al. 2006) and characterized by gene expression analysis (Reddien et al. 2005; Oviedo and Levin 2007), whereas ultrastructural features can provide more details about different neoblast subpopulations (Hayashi et al. 2006; Higuchi et al. 2007).

The effects of drug compounds on cell behavior can be conveniently tested in planaria, because regeneration is a sensitive assay for changes in proliferation, migration, differentiation, and morphogenetic cues. Additionally, diverse physiological processes can be analyzed in the whole organism in real time (e.g., RNAi followed by imaging of physiological parameters; see Live Imaging of Planarian Membrane Potential Using DiBAC $_{4}$ (3) [Oviedo et al. 2008c]), providing an opportunity to dissect complex molecular interactions in vivo. Also, because regeneration can reveal subtle changes in cell signaling, planarians are a good system in which to identify and molecularly characterize the effects of weak electromagnetic stimulation (Novikov et al. 2002).

Protocols for running behavioral experiments have been refined over the years since planarians were first used for behavioral work (McConnell 1965). Although these experiments can be performed manually, the future of this field clearly rests in automated systems for training and testing worms, both for high-throughput approaches and for establishing basic quantitative and objective results. The construction and use of such automated systems has most recently been described by Hicks et al. (2006).

\section{ACKNOWLEDGMENTS}

We thank Dr. W. Beane for comments and suggestions on the manuscript. N.J.O. is a National Institutes of Health (NIH) fellow supported under the Ruth L. Kirschstein National Research Service Award (F32 GM078774). D.S.A. is supported by the National Institute of Dental and Craniofacial Research (NIDCR) grant 1K22-DE016633. M.L. is supported by the National Science Foundation (NSF) grant IBN 0347295, National Highway Traffic Safety Administration (NHTSA) grant DTNH2206-G-00001, and NIH grant R21 HD055850. This review was prepared in a Forsyth Institute facility renovated with support from Research Facilities Improvement Grant Number CO6RR11244 from the National Center for Research Resources, NIH. We apologize to our colleagues whose work we could not cite due to space limitations.

\section{REFERENCES}

Agata, K. 2003. Regeneration and gene regulation in planarians. Curr. Opin. Genet. Dev. 13: 492-496.

Baguñà, J. 1999. From morphology and karyology to molecules. New methods for taxonomical identification of asexual populations of freshwater planarians. A tribute to Professor Mario Benazzi. Ital. J. Zool. 66: 207-214.

Baguñà, J. and Romero, R. 1981. Quantitative analysis of cell types during growth, degrowth and regeneration in the planarians Dugesia mediterranea and Dugesia tigrina. Hydrobiologia 84: 181194.

Benazzi, M., Baguñà, J., and Ballester, R. 1970. First report on an asexual form of the planarian Dugesia lugubris s.l. Rend. Acc. Naz. Lincei 48: 282-284.

Benazzi, M., Ballester, R., Baguñà, J., and Puccinelli, I. 1972. The fissiparous race of the planarian Dugesia lugubris S.L. found in Barcelona (Spain) belongs to the biotype g: Comparative analysis of the karyotypes. Caryologia 25: 59-68.

Benazzi, M., Baguñà, J., Ballester, R., Puccinelli, I., and Del Papa, R.
1975. Further contribution to the taxonomy of the "Dugesia lugubris-polychroa group" with description of Dugesia mediterranea n.sp. (Tricladida, Paludicola). Boll. Zool. 42: 81-89.

Cebrià, F. 2007. Regenerating the central nervous system: How easy for planarians!. Dev. Genes Evol. 217: 733-748.

De Vries, E. 1985. The biogeography of the genus Dugesia (Turbellaria, Tricladida, Paludicola) in the Mediterranean region. J. Biogeogr. 12: 509-518.

Handberg-Thorsager, M. and Saló, E. 2007. The planarian nanos-like gene Smednos is expressed in germline and eye precursor cells during development and regeneration. Dev. Genes Evol. 217: 403-411.

Hayashi, T., Asami, M., Higuchi, S., Shibata, N., and Agata, K. 2006. Isolation of planarian X-ray-sensitive stem cells by fluorescenceactivated cell sorting. Dev. Growth Differ. 48: 371-380.

Hicks, C., Sorocco, D., and Levin, M. 2006. Automated analysis of behavior: A computer-controlled system for drug screening and the investigation of learning. J. Neurobiol. 66: 977-990. 
Higuchi, S., Hayashi, T., Hori, I., Shibata, N., Sakamoto, H., and Agata, K. 2007. Characterization and categorization of fluorescence activated cell sorted planarian stem cells by ultrastructural analysis. Dev. Growth Differ. 49: 571-581.

McConnell, J.V., ed. 1965. A manual of psychological experimentation on planarians. Worm Runner's Digest, Ann Arbor, MI.

McKay, R.D. 2004. Stem cell biology and neurodegenerative disease. Philos. Trans. R. Soc. Lond. B Biol. Sci. 359: 851-856.

Morgan, T.H. 1898. Experimental studies of the regeneration of Planaria maculata. Arch. Entw. Mech. Org. 7: 364-397.

Newmark, P.A. and Sánchez Alvarado, A. 2000. Bromodeoxyuridine specifically labels the regenerative stem cells of planarians. Dev. Biol. 220: 142-153.

Newmark, P.A. and Sánchez Alvarado, A. 2002. Not your father's planarian: A classic model enters the era of functional genomics. Nat. Rev. Genet. 3: 210-219.

Nicolas, C., Abramson, C., and Levin, M. 2008. Analysis of behavior in the planarian model. In Planaria: A model for drug action and abuse (ed. R.B. Raffa), RG Landes, Austin, TX.

Nogi, T. and Levin, M. 2005. Characterization of innexin gene expression and functional roles of gap-junctional communication in planarian regeneration. Dev. Biol. 287: 314-335.

Novikov, V.V., Sheiman, I.M., Lisitsyn, A.S., Klyubin, A., and Fesenko, E.E. 2002. Dependence of the effect of weak combined low-frequency variable and constant magnetic fields on the intensity of asexual reproduction of planarians Dugesia tigrina on the magnitude of the variable field. Biofizika 47: 564-567.

Ogawa, K., Wakayama, A., Kunisada, T., Orii, H., Watanabe, K., and Agata, K. 1998. Identification of a receptor tyrosine kinase involved in germ cell differentiation in planarians. Biochem. Biophys. Res. Commun. 248: 204-209.

Orii, H., Sakurai, T., and Watanabe, K. 2005. Distribution of the stem cells (neoblasts) in the planarian Dugesia japonica. Dev. Genes Evol. 215: 143-157.

Oviedo, N.J. and Levin, M. 2007. smedinx-11 is a planarian stem cell gap junction gene required for regeneration and homeostasis. Development 134: 3121-3131.

Oviedo, N.J. and Levin, M. 2008. The planarian regeneration model as a context for the study of drug effects and mechanisms. In Planaria: A model for drug action and abuse (ed. R.B. Raffa), RG Landes, Austin, TX.

Oviedo, N.J., Newmark, P.A., and Sánchez Alvarado, A. 2003. Allometric scaling and proportion regulation in the freshwater planarian Schmidtea mediterranea. Dev. Dyn. 226: 326-333.

Oviedo, N.J., Nicolas, C.L., Adams, D.S., and Levin, M. 2008a. Establishing and maintaining a colony of planarians. Cold Spring Harb. Protoc. (this issue). doi: 10.1101/pdb.prot5053.

Oviedo, N.J., Nicolas, C.L., Adams, D.S., and Levin, M. 2008b. Gene knockdown in planarians using RNA interference. Cold Spring Harb. Protoc. (this issue). doi: 10.1101/pdb.prot5054.

Oviedo, N.J., Nicolas, C.L., Adams, D.S., and Levin, M. 2008c. Live imaging of planarian membrane potential using $\operatorname{DiBAC}_{4}(3)$. Cold Spring Harb. Protoc. (this issue). doi: 10.1101/pdb.prot5055.

Oviedo, N.J., Pearson, B., Levin, M., and Sánchez Alvardo, A. 2008d. Planarian PTEN homologs regulate stem cells and regeneration through TOR signaling. Dis. Mod. Mech. doi: 10.1242/ dmm.000117.
Pellettieri, J. and Sánchez Alvarado, A. 2007. Cell turnover and adult tissue homeostasis: From humans to planarians. Annu. Rev. Genet. 41: 83-105.

Randolph, H. 1897. Observations and experiments on regeneration in planarians. Arch. Entw. Mech. Org. 5: 352-372.

Reddien, P.W. and Sánchez Alvarado, A. 2004. Fundamentals of planarian regeneration. Annu. Rev. Cell Dev. Biol. 20: 725-757.

Reddien, P.W., Oviedo, N.J., Jennings, J.R., Jenkin, J.C., and Sánchez Alvarado, A. 2005. SMEDWI-2 is a PIWI-like protein that regulates planarian stem cells. Science 310: 1327-1330.

Reddien, P.W., Newmark, P.A., and Sánchez Alvarado, A. 2008. Gene nomenclature guidelines for the planarian Schmidtea mediterranea. Dev. Dyn. doi: 10.1002/dvdy.21623.

Robb, S.M., Ross, E., and Sánchez Alvarado, A. 2008. SmedGD: The Schmidtea mediterranea genome database. Nucleic Acids Res. 36: D599-D606.

Rossi, L., Salvetti, A., Lena, A., Batistoni, R., Deri, P., Pugliesi, C., Loreti, E., and Gremigni, V. 2006. DjPiwi-1, a member of the PAZPiwi gene family, defines a subpopulation of planarian stem cells. Dev. Genes Evol. 216: 335-346.

Rossi, L., Salvetti, A., Batistoni, R., Deri, P., and Gremigni, V. 2007 a. Planarians, a tale of stem cells. Cell. Mol. Life Sci. 65: 16-23.

Rossi, L., Salvetti, A., Marincola, F.M., Lena, A., Deri, P., Mannini, L., Batistoni, R., Wang, E., and Gremigni, V. 2007b. Deciphering the molecular machinery of stem cells: A look at the neoblast gene expression profile. Genome Biol. 8: R62 doi: 10.1186/gb-2007-84-r62.

Sánchez Alvarado, A. 2003. The freshwater planarian Schmidtea mediterranea: Embryogenesis, stem cells and regeneration. Curr. Opin. Genet. Dev. 13: 438-444.

Sánchez Alvarado, A. 2006. Planarian regeneration: Its end is its beginning. Cell 124: 241-245.

Sánchez Alvarado, A. 2007. Stem cells and the planarian Schmidtea mediterranea. C. R. Biol. 330: 498-503.

Sánchez Alvarado, A. and Kang, H. 2005. Multicellularity, stem cells, and the neoblasts of the planarian Schmidtea mediterranea. Exp. Cell Res. 306: 299-308.

Sánchez Alvarado, A., Newmark, P.A., Robb, S.M., and Juste, R. 2002. The Schmidtea mediterranea database as a molecular resource for studying platyhelminthes, stem cells and regeneration. Development 129: 5659-5665.

Sato, K., Shibata, N., Orii, H., Amikura, R., Sakurai, T., Agata, K., Kobayashi, S., and Watanabe, K. 2006. Identification and origin of the germline stem cells as revealed by the expression of nanosrelated gene in planarians. Dev. Growth Differ. 48: 615-628.

Umesono, Y., Watanabe, K., and Agata, K. 1997. A planarian orthopedic homolog is specifically expressed in the branch region of both the mature and regenerating brain. Dev. Growth Differ. 39: 723-727.

Wang, Y., Zayas, R.M., Guo, T., and Newmark, P.A. 2007. nanos function is essential for development and regeneration of planarian germ cells. Proc. Natl. Acad. Sci. 104: 5901-5906.

Zayas, R.M., Hernandez, A., Habermann, B., Wang, Y., Stary, J.M., and Newmark, P.A. 2005. The planarian Schmidtea mediterranea as a model for epigenetic germ-cell specification: Analysis of ESTs from the hermaphroditic strain. Proc. Natl. Acad. Sci. 102: 1849118496. 


\section{Planarians: A Versatile and Powerful Model System for Molecular Studies of Regeneration, Adult Stem Cell Regulation, Aging, and Behavior}

Néstor J. Oviedo, Cindy L. Nicolas, Dany S. Adams and Michael Levin

Cold Spring Harb Protoc; doi: 10.1101/pdb.emo101

\begin{tabular}{rc}
\hline $\begin{array}{r}\text { Email Alerting } \\
\text { Service }\end{array}$ & Receive free email alerts when new articles cite this article - click here. \\
\hline $\begin{array}{c}\text { Subject } \\
\text { Categories }\end{array}$ & $\begin{array}{c}\text { Browse articles on similar topics from Cold Spring Harbor Protocols. } \\
\text { Developmental Biology (728 articles) } \\
\text { Emerging Model Organisms (321 articles) } \\
\text { Laboratory Organisms, general (923 articles) }\end{array}$ \\
\hline
\end{tabular}

